\title{
Disruption of lipid metabolism in the liver of the pregnant rat fed folate- deficient and methyl donor-deficient diets
}

\author{
Christopher J. McNeil, Susan M. Hay, Garry J. Rucklidge, Martin Reid, Gary Duncan, \\ Christopher A. Maloney and William D. Rees* \\ The Rowett Research Institute, Greenburn Road, Bucksburn, Aberdeen, AB21 9SB, Scotland
}

(Received 2 February 2007 - Revised 22 May 2007 - Accepted 15 June 2007)

\begin{abstract}
The importance of folic acid and the methionine cycle in fetal development is well recognised even though the mechanism has not been established. Since the cycle is active in the maternal liver, poor folate status may modify hepatic metabolism. Pregnant rats were fed diets deficient in folic acid (-F) or in three key methyl donors, folic acid, choline and methionine (-FLMLC) and the maternal liver was analysed on day 21 of gestation. Two-dimensional gel electrophoresis of soluble proteins identified differentially abundant proteins, which could be allocated into nine functional groups. Five involved in metabolic processes, namely, folate/methionine cycle, tyrosine metabolism, protein metabolism, energy metabolism and lipid metabolism, and three in cellular processes, namely, endoplasmic reticulum function, bile production and antioxidant defence. The mRNA for sterol regulatory element-binding protein- $1 \mathrm{c}$ and acetyl-CoA carboxylase-1 (fatty acid synthesis) were decreased by both $-\mathrm{F}$ and FLMLC diets. The mRNA for PPAR $\alpha$ and PPAR $\gamma$ and carnitine palmitoyl transferase (fatty acid oxidation) were increased in the animals fed the -FLMLC diets. Changes in the abundance of proteins associated with intracellular lipid transport suggest that folate deficiency interferes with lipid export. Reduced fatty acid synthesis appeared to prevent steatosis in animals fed the $-\mathrm{F}$ diet. Even with increased oxidation, TAG concentrations were approximately three-fold higher in animals fed the -FLMLC diet and were associated with an increase in the relative abundance of proteins associated with oxidative stress. Fetal development may be indirectly affected by these changes in hepatic lipid metabolism.
\end{abstract}

Methionine: Choline: Folic acid: Endoplasmic reticulum

There is overwhelming evidence to show that folic acid supplements during pregnancy are efficacious in reducing the risk of neural tube defects (NTD) in the fetus. However, numerous studies of folate levels have failed to establish a connection between folic acid metabolism in the fetus and the risk of NTD ${ }^{1,2}$. Moreover, the frequency of NTD is not increased following the inactivation of enzymes essential for folate metabolism in the fetus ${ }^{3}$. An adequate intake of folic acid during the weeks just before and after conception also improves the overall outcome ${ }^{4}$, notably, increasing infant birth weight ${ }^{5}$ and reducing the incidence of spontaneous abortion and pre-term delivery ${ }^{6}$. However, as with NTD, there is no clear evidence to link impaired fetal growth directly to folate deficiency in fetal tissues. These observations suggest that some of the broad benefits of improved folate status could be the indirect consequences of improvements in maternal metabolism.

Derivatives of folic acid play an important role in onecarbon transfer reactions, supplying methyl groups to complete the methionine cycle. Under some circumstances choline and methionine also donate methyl groups and their availability in the diet modifies the requirement for folic acid in one-carbon transfers. Before conception, the main consumer of methyl groups supplied by the methionine cycle is believed to be the hepatic enzyme phosphatidyl ethanolamine methyl transferase (PEMT) ${ }^{7}$. Therefore, folate deficiency during pregnancy may alter hepatic function and disturb maternal metabolic homeostasis, indirectly affecting fetal development. In the present study, a combination of two approaches has been used to examine the liver of pregnant rats fed one of two experimental diets; either a diet deficient in folic acid $(-\mathrm{F})$, or one deficient in all three key methyl donors, folic acid, choline and methionine (-FLMLC). First, we analysed the soluble protein fraction from the maternal liver on day 21 of gestation (term is $22.5 \mathrm{~d}$ in the rat) by two-dimensional electrophoresis. Since the cytosol is in communication with many of the intracellular compartments, this fraction gives an overall picture of the changes. Second, we carried out a targeted analysis of hepatic mRNA coding for the enzymes that control the synthesis of fatty acids (acetyl CoA carboxylase-1), fatty acid oxidation (carnitine palmitoyl transferase-1) and the transcriptional regulators that control them (PPAR, CCAAT enhancer binding protein and sterol response element-binding protein-1c (SREBP-1c)). We have also investigated the expression of gadd153 and p57 to evaluate the extent of metabolic stress.

Abbreviations: -F diet, diet deficient in folic acid; -FLMLC diet, diet deficient in all three key methyl donors, folic acid, choline and methionine; NTD, neural tube defect; PEMT, phosphatidyl ethanolamine methyl transferase; SREBP-1c, sterol response element binding protein-1c.

* Corresponding author: William D. Rees, fax +44 1224 716622, email wdr@rri.sari.ac.uk 


\section{Methods}

\section{Experimental diets}

The experimental diets were based on the AIN-76 formula ${ }^{8}$ and contained $90 \mathrm{~g}$ casein $/ \mathrm{kg}$ supplemented with a mixture of synthetic amino acids equivalent to those found in a further $90 \mathrm{~g} / \mathrm{kg}$ casein as described previously ${ }^{9}$. Folic acid was omitted from the $-\mathrm{F}$ and -FLMLC diets. No additional methionine was added to the -FLMLC diet (total methionine concentration $2.3 \mathrm{mg}$ methionine per $\mathrm{kg}$ diet compared with $5.6 \mathrm{mg}$ methionine per $\mathrm{kg}$ diet in the control) and choline chloride was reduced to $0.1 \% \mathrm{w} / \mathrm{w}$ (compared with $0.2 \%$ $\mathrm{w} / \mathrm{w}$ in the control).

\section{Animals}

All experimental procedures were approved by the ethical review committee of the Rowett Research Institute and conducted in accordance with the UK Animals (Scientific Procedures) Act, 1986. Female rats of the Rowett Hooded strain bred in the institute were allocated to three groups of eight animals. The animals were approximately 8 to 10 weeks of age at the start of the experiment with a mean body weight of $208 \mathrm{~g}$. There were no significant differences between the weights of animals in the different treatment groups when the experiment commenced. Animals were housed on sawdust bedding and no steps were taken to prevent coprophagy. Three groups of animals were fed control, $-\mathrm{F}$ or -FLMLC diets. The animals were offered the experimental diets for a 2-week adaptation period and then were mated with males of the same strain. The day on which a vaginal plug was detected was denoted day 0 . The female rats were maintained on their corresponding diets until day 21 of gestation, when they were anaesthetised by halothane inhalation and killed by exsanguination. The fetuses were rapidly removed, weighed and killed by decapitation. Samples of the maternal liver were frozen in liquid $\mathrm{N}$ as soon as possible and subsequently stored at $-80^{\circ} \mathrm{C}$ until required. Plasma was prepared from samples of trunk blood and alanine aminotransferase and aspartate aminotransferase were analysed using a Konelab selective chemistry analyser (Labmedics Ltd, Salford, Manchester, UK).

\section{Proteomics}

Pieces of frozen maternal liver from six pregnant animals chosen randomly from each group were homogenised in buffer containing protease inhibitors as described previously ${ }^{10}$. The homogenate was centrifuged for $30 \mathrm{~min}$ at $100000 \mathrm{~g}$ at $4^{\circ} \mathrm{C}$ (Beckman TL-100 centrifuge; Beckman Coulter Ltd, High Wycombe, Bucks, UK). Proteins in the supernatant $(300 \mu \mathrm{g}$ from each extract) were separated in the first dimension on BioRad immobilized $\mathrm{pH}$ gradient strips (pI 3-10; BioRad, Hemel Hempstead, UK) ${ }^{11}$. Following equilibration with the second dimension buffer, which contained $135 \mathrm{~mm}$-iodoacetamide, proteins were separated on an $18 \times 18 \mathrm{~cm}$ SDS polyacrylamide gel at $200 \mathrm{~V}$ for $9.5 \mathrm{~h}^{11}$. Molecular weights were determined with a BioRad Precision Plus Protein Mr standard. Gels were stained with colloidal coomassie brilliant blue, dried and scanned as previously described ${ }^{11}$. The gel images were analysed (PDQuest v7 BioRad) and spots of interest were excised from the gels using a robotic spot cutter (BioRad). Protein identities were determined by MALDI-TOF (Voyager-DE PRO; Applied Biosystems, Warrington, Cheshire, UK) or by liquid chromatography/MS/MS (Q-trap; Applied Biosystems) MS of trypsin-digested protein spots as previously described $^{11}$. The mass spectra of spots that were significantly different were corrected for isotope abundance and the resulting peptide mass list profiles were analysed using the Matrix Science 'Mascot' web tool (http://www. matrixscience.com). The Mascot database search criteria allowed one missed cleavage, carbamidomethyl modification of cysteine; partial oxidation of methionine, a charged state of $\mathrm{MH}+$ and positive identities of at least $20 \%$ matched peptides covering at least $10 \%$ of the protein sequence. Positive matches with proteins in the database were assigned at a probability value of $P<0.05$.

\section{TAG analysis}

Approximately $0.2 \mathrm{~g}$ tissue was homogenised in $1 \mathrm{ml}$ ice cold $0.145 \mathrm{M}-\mathrm{NaCl}$. The homogenate was extracted with $10 \mathrm{ml}$ chloroform:methanol (2:1). The chloroform phase was transferred to a clean tube and the residue was extracted with a further $3 \mathrm{ml}$ chloroform. The extracts were combined, the chloroform was removed by evaporation and the lipids were dissolved in $5 \mathrm{ml}$ absolute ethanol. TAG in $10 \mu \mathrm{l}$ samples of the ethanol suspension were measured by adding $0.25 \mathrm{ml}$ reagent (ThemoElectron Triglyceride Kit; Lab Medics Ltd), incubating for $20 \mathrm{~min}$ at room temperature and measuring the absorbance at $510 \mathrm{~nm}$. TAG concentrations in the samples were determined from a standard curve.

\section{Real Time PCR}

Total RNA was extracted using the Trizol reagent (Sigma, Poole, Dorset, UK) as described previously ${ }^{12}$. Samples of $50 \mathrm{ng}$ total RNA were reverse transcribed using the TaqMan Reverse Transcription Reagents Kit (Applied Biosystems) primed with random hexamers. The levels of cDNA relative to the $18 \mathrm{~S}$ ribosomal RNA were measured using the SYBR Green real time PCR kit (Applied Biosystems). The primer sequences used are described in Table 1 . The identity of the products was confirmed by sequencing and the response was linear over the range measured. Relative target quantity was calculated from the standard curve and the results expressed as the ratio of the product relative to the product from the $18 \mathrm{~S}$ rRNA.

\section{Statistical analysis}

Data are presented as means with their standard errors of the mean. Scans of the proteomic gels (six animals per treatment) were normalised using PDQuest software to determine the mean corrected spot density. The PDQuest statistical analysis (Student's $t$ test) was performed by pairwise comparisons of each spot on the control gels with either the $\mathrm{F}$ or the -FLMLC gels. The spot densities of proteins identified in this preliminary screen were subsequently analysed by one-way ANOVA followed by Fischer's multiple comparison test (Genstat 7 statistical package; Lawes Agricultural Trust, Rothamsted Experimental Station, Harpenden, 
Table 1. Primers used for PCR analysis*

\begin{tabular}{lllc}
\hline Gene & \multicolumn{1}{c}{ Forward primer } & \multicolumn{1}{c}{ Reverse primer } & Product size \\
\hline Acc & CAACGCCTTCACACCACCTT & AGCCCATTACTTCATCAAAGATCCT & 104 \\
L-CPT-1 & CGGTTCAAGAATGGCATCATC & TCACACCCACCACCACGATA & 75 \\
PPAR- $\alpha$ & CTATGGAGTCCACGCATGTGA & TACGCCAGCTTTAGCCGAAT & 68 \\
PPAR $\gamma$ & CACAATGCCATCAGGTTTGG & CAGCTTCTCCTTCTCGGCCT & 50 \\
SREBP-1c & GGAGCCATGGATTGCACATT & CAAATAGGCCAGGGAAGTCAC & 75 \\
C/EBP- $\alpha$ & TGCGCAAGAGCCGAGATAA & TGGTCAACTCCAACACCTTCTG & 69 \\
Gadd153 & CCACCACACCTGAAAGCAGAA & CACTGTCTCAAAGGCGAAAGG & 78 \\
p57 & CCGGTTCCTGCTACATGAATG & GCAGCGGACGATGGAAGA & 64 \\
$18 \mathrm{~s}$ & CATTCGTATTGCGCCGCTA & ATGCTTTCGCTCTGGTTCGT & 66 \\
\hline
\end{tabular}

${ }^{*}$ For details of procedures, see Methods.

Acc, acetyl CoA carboxylase; L-CPT-1, carnitine palmitoyl transferase; SREBP-1c, sterol response element-binding protein $1 \mathrm{c} ; \mathrm{C} / \mathrm{EBP}-\alpha, \mathrm{CCAAT}$ enhancer binding protein $\alpha$.

Herts., UK). The gene expression data were analysed by one-way ANOVA followed by Fischer's multiple comparison test (Genstat).

\section{Results}

\section{Maternal growth}

The growth of the animals has been described previously'. Briefly, the weight gain of animals fed the $-\mathrm{F}$ diet during gestation was not different from the control group, whereas the animals fed the -FLMLC diet gained approximately $15 \%$ less than animals in the control group. Food intake during the last week of gestation did not differ significantly between the groups. The number of fetuses was not affected by the diets. Fetuses of dams fed the $-\mathrm{F}$ diet were approximately $18 \%$ heavier (4.84 (SEM 0.07) g) than the controls (4.10 (SEM 0.03) g), while those from dams fed the FLMLC diet were approximately $10 \%$ smaller than the controls (3.71 (SEM 0.07) g). There were no gross abnormalities in either restricted group. The livers of dams in the $-\mathrm{F}$ group (10.1 (SEM 0.6) g) and -FLMLC group (9.1 (SEM 0.4) g) were significantly smaller $(P=0.002)$ than those of animals in the control group (11.8 (SEM 1.2) g). The metabolic changes observed in these animals have been described previously ${ }^{9}$. Hepatic folate was reduced by approximately $80 \%$ in animals fed -F or -FLMLC diets. Hepatic phosphocholine stores were reduced by approximately $30 \%(-\mathrm{F})$ and $60 \%$ (-FLMLC) when compared with the control. Plasma homocysteine, glycine, serine and threonine concentrations in the maternal plasma of animals fed the $-\mathrm{F}$ diet were higher than the control and this was further exacerbated by the -FLMLC $\operatorname{diet}^{9}$.

\section{Proteomic analysis of soluble proteins}

Two-dimensional gel electrophoresis separated approximately 900 proteins in the soluble fraction of the female rat liver at day 21 of gestation. Comparisons of the relative abundance of proteins from the liver of animals fed the control diet with those fed the $-\mathrm{F}$ diet showed that eighteen proteins were up regulated and sixteen were down regulated (Student's $t$ test $P<0 \cdot 05$ ). Comparisons of the patterns from animals fed the -FLMLC diet with the control showed that twenty-six proteins were up regulated and sixteen proteins were down regulated $(P<0 \cdot 05)$. A further thirteen proteins were identified as changed $(P<0.05)$ in a comparison of the soluble hepatic proteins in livers from animals fed $-\mathrm{F}$ and $-\mathrm{F}$ M LC diets.

All of the proteins identified in the initial analysis were sequenced by MS and their identities are shown in Table 2 . The proteins were allocated to different groups depending on their functions. Proteins associated with metabolic functions are shown in Table 3. They include proteins associated with folate/methionine metabolism, protein and amino acid metabolism, tyrosine metabolism, energy metabolism and lipid metabolism. There were also a number of proteins associated with cellular functions and these are shown in Table 4. These proteins could be allocated into groups associated with the endoplasmic reticulum, bile production and oxidative stress. There were also a small number of proteins that could not be allocated to specific functions.

\section{TAG in the maternal liver}

There were no differences in maternal serum TAG concentrations (Fig. 1). The TAG content of the maternal liver of animals fed $-\mathrm{F}$ diets was not significantly different from the controls. In contrast, the hepatic TAG content of dams fed the -FLMLC diet was increased by three-fold compared with the controls. The serum alanine aminotransferase and aspartate aminotransferase activities were not different from the controls in either $-\mathrm{F}$ or -FLMLC diet groups (data not shown).

\section{Gene expression in the maternal liver}

The relative expression of a number of mRNA associated with lipid metabolism in the maternal liver is shown in Table 5. The hepatic acetyl CoA carboxylase-1 mRNA levels was decreased by approximately $25 \%$ in the livers of animals fed the $-\mathrm{F}$ and -FLMLC diets when compared with the controls. At the same time, there was an increase of approximately $50 \%$ in the relative expression of carnitine palmitoyl transferase- 1 in animals fed the -FLMLC diet. The mRNA for PPAR $\alpha$ and PPAR $\gamma$ was increased by approximately $25 \%$ in the livers of animals fed the -FLMLC diet. The mRNA for SREBP-1c was reduced by approximately $50 \%$ in the animals fed $-\mathrm{F}$ and -FLMLC diets. There was no change in the relative expression of CCAAT enhancer binding protein- $\alpha$, gadd 153 or p57. 
Table 2. Sizes and identities of differentially abundant proteins $\dagger$

\begin{tabular}{|c|c|c|c|c|c|c|c|}
\hline PI number & $\mathrm{p}$ (peptide mass fingerprint MALDI) & p (Ion Score, LCMSMS) & Entrez code & $\mathrm{Mr}$ & Predicted Mr & $\mathrm{pl}$ & Predicted pl \\
\hline 206 & & $1 \cdot 1 \mathrm{E}-15$ & BAA04261 & $28 \cdot 9$ & $28 \cdot 6$ & 5.5 & $4 \cdot 8$ \\
\hline 606 & & $2 \cdot 0 \mathrm{E}-04$ & AAH62395 & $52 \cdot 9$ & $48 \cdot 1$ & $5 \cdot 1$ & $4 \cdot 3$ \\
\hline 1114 & & $3 \cdot 8 \mathrm{E}-13$ & A57716 & 21.9 & 21.9 & 5.9 & $5 \cdot 3$ \\
\hline $1306^{*}$ & 0.05 & $2 \cdot 1 \mathrm{E}-07$ & AAH86581 & $32 \cdot 0$ & $35 \cdot 8$ & $5 \cdot 8$ & $5 \cdot 2$ \\
\hline 1308 & & $1 \cdot 6 \mathrm{E}-27$ & BAA90692 & 33.5 & 33.9 & $5 \cdot 9$ & $5 \cdot 3$ \\
\hline $1509^{*}$ & 0.00069 & & AAH60597 & $48 \cdot 4$ & $50 \cdot 2$ & $5 \cdot 7$ & $4 \cdot 8$ \\
\hline 2005 & & $7 \cdot 7 \mathrm{E}-14$ & AAD47293 & 14.9 & $15 \cdot 8$ & $6 \cdot 1$ & $5 \cdot 1$ \\
\hline 2203 & & & AAH78867 & $29 \cdot 1$ & 30.9 & $6 \cdot 2$ & $5 \cdot 4$ \\
\hline 2401 & & $2 \cdot 8 \mathrm{E}-17$ & Q63041 & $38 \cdot 7$ & $55 \cdot 1$ & $6 \cdot 0$ & 4.9 \\
\hline 2407 & $3 \cdot 80 \mathrm{E}-06$ & & CAA06313 & $42 \cdot 0$ & $39 \cdot 3$ & $6 \cdot 1$ & $5 \cdot 5$ \\
\hline 2510 & $2 \cdot 00 \mathrm{E}-03$ & & AAH63812 & $46 \cdot 5$ & $46 \cdot 4$ & $6 \cdot 1$ & $5 \cdot 3$ \\
\hline $2713^{*}$ & 0.0052 & $1 \cdot 5 \mathrm{E}-41$ & CAA60040 & $66 \cdot 6$ & $76 \cdot 0$ & $6 \cdot 3$ & $5 \cdot 4$ \\
\hline $2714^{*}$ & 0.00042 & $5 \cdot 9 \mathrm{E}-65$ & AAB34982 & $74 \cdot 8$ & 74.0 & $6 \cdot 3$ & $5 \cdot 9$ \\
\hline 2815 & & $4 \cdot 5 \mathrm{E}-41$ & AAH60518 & $94 \cdot 3$ & $90 \cdot 0$ & $6 \cdot 2$ & $6 \cdot 1$ \\
\hline 3002 & & $5 \cdot 1 \mathrm{E}-14$ & CAA85429 & $14 \cdot 1$ & $13 \cdot 2$ & 6.5 & $6 \cdot 1$ \\
\hline 3607 & & $2 \cdot 2 \mathrm{E}-31$ & P17425 & 53.5 & $58 \cdot 0$ & 6.5 & $\#$ \\
\hline $3716^{*}$ & 1.00E-06 & $5 \cdot 7 \mathrm{E}-18$ & AAH88347 & 74.9 & $72 \cdot 7$ & $6 \cdot 7$ & $5 \cdot 8$ \\
\hline 4002 & & 1.5E-05 & P02632 & $13 \cdot 1$ & $14 \cdot 3$ & 6.7 & $7 \cdot 8$ \\
\hline 4106 & & $1 \cdot 4 \mathrm{E}-07$ & AAA42020 & $19 \cdot 6$ & $20 \cdot 1$ & $7 \cdot 0$ & $5 \cdot 7$ \\
\hline 4306 & & $5 \cdot 8 \mathrm{E}-22$ & AAC64180 & $34 \cdot 0$ & $36 \cdot 6$ & 6.9 & $6 \cdot 2$ \\
\hline $4404^{*}$ & 8.50E-06 & $2 \cdot 9 \mathrm{E}-19$ & AAH78930 & $42 \cdot 7$ & $45 \cdot 8$ & 6.9 & 6.0 \\
\hline 4405 & & $2 \cdot 8 \mathrm{E}-10$ & Q91VW4 & 39.5 & 40.6 & 6.9 & $6 \cdot 2$ \\
\hline $4501^{*}$ & 0.043 & $2 \cdot 7 \mathrm{E}-34$ & AAH89770 & $48 \cdot 8$ & $44 \cdot 1$ & $6 \cdot 8$ & $5 \cdot 7$ \\
\hline $4703^{*}$ & 0.0072 & $3 \cdot 5 E-23$ & AAH61765 & 64.5 & $59 \cdot 1$ & $6 \cdot 8$ & $5 \cdot 9$ \\
\hline $4707^{*}$ & 1.60E-05 & $3 \cdot 7 E-45$ & CAA24532 & $69 \cdot 2$ & 70.7 & $7 \cdot 0$ & $6 \cdot 1$ \\
\hline $4801^{*}$ & 0.00042 & & BAB16378 & 119.9 & $124 \cdot 2$ & $6 \cdot 8$ & $5 \cdot 5$ \\
\hline 4809 & & $4 \cdot 4 \mathrm{E}-28$ & AAH89101 & $100 \cdot 0$ & $99 \cdot 7$ & $7 \cdot 0$ & $5 \cdot 6$ \\
\hline $4904^{*}$ & 0.0049 & $6 \cdot 9 E-53$ & AAB59717 & $157 \cdot 0$ & $165 \cdot 7$ & $7 \cdot 0$ & $6 \cdot 3$ \\
\hline 5008 & & 1.4E-09 & P02692 & $12 \cdot 9$ & $14 \cdot 2$ & $7 \cdot 3$ & $6 \cdot 7$ \\
\hline 5205 & & $6 \cdot 1 \mathrm{E}-05$ & P41034 & $29 \cdot 4$ & 31.9 & $7 \cdot 2$ & 6.5 \\
\hline 5208 & & $3 \cdot 1 \mathrm{E}-10$ & P22789 & $27 \cdot 8$ & 33.2 & $7 \cdot 4$ & $7 \cdot 6$ \\
\hline 5311 & & $8 \cdot 6 \mathrm{E}-19$ & Q920P0 & 31.0 & $25 \cdot 7$ & $7 \cdot 3$ & $6 \cdot 8$ \\
\hline 5314 & & $2 \cdot 3 \mathrm{E}-10$ & Q5BKC8 & $34 \cdot 1$ & 37.5 & $7 \cdot 4$ & $6 \cdot 7$ \\
\hline 5410 & & $7 \cdot 4 \mathrm{E}-40$ & O88655 & $73 \cdot 2$ & 74.5 & $7 \cdot 3$ & $6 \cdot 3$ \\
\hline $5413^{*}$ & 0.0015 & $2 \cdot 1 \mathrm{E}-24$ & AAH76381 & 41.7 & $46 \cdot 2$ & $7 \cdot 3$ & $6 \cdot 7$ \\
\hline $5602^{*}$ & $1 \cdot 20 \mathrm{E}-05$ & $1 \cdot 1 \mathrm{E}-46$ & $\mathrm{AAH} 79381$ & $56 \cdot 4$ & $56 \cdot 5$ & $7 \cdot 1$ & $6 \cdot 8$ \\
\hline $5607^{*}$ & 0.003 & $1 \cdot 6 \mathrm{E}-19$ & AAH81884 & 52.9 & $58 \cdot 1$ & $7 \cdot 2$ & 6.6 \\
\hline 5612 & & $8 \cdot 6 \mathrm{E}-22$ & AAH62069 & 57.4 & 61.4 & $7 \cdot 4$ & $6 \cdot 1$ \\
\hline 5614 & & $5 \cdot 1 \mathrm{E}-15$ & NP036756 & 55.4 & $59 \cdot 0$ & $7 \cdot 2$ & $6 \cdot 2$ \\
\hline $5711^{*}$ & 0.002 & 2.7E-39 & AAH99807 & 62.9 & 63.5 & $7 \cdot 3$ & $6 \cdot 2$ \\
\hline 5801 & $1.00 \mathrm{E}-10$ & & AAH85697 & $87 \cdot 7$ & $96 \cdot 2$ & $7 \cdot 1$ & $6 \cdot 8$ \\
\hline $6408^{*}$ & 0.00074 & $1 \cdot 1 \mathrm{E}-25$ & $1 R L A B$ & $39 \cdot 3$ & $34 \cdot 1$ & $7 \cdot 7$ & $6 \cdot 2$ \\
\hline 6506 & & 8.7E-25 & P10760 & $45 \cdot 1$ & 43.2 & $7 \cdot 6$ & 6.4 \\
\hline $6508^{*}$ & 0.0013 & $4 \cdot 2 \mathrm{E}-12$ & AAH78948 & $45 \cdot 5$ & $50 \cdot 7$ & $7 \cdot 7$ & 6.7 \\
\hline $6709^{*}$ & $1 \cdot 20 \mathrm{E}-05$ & $6 \cdot 1 \mathrm{E}-40$ & AAB42378 & $61 \cdot 1$ & $59 \cdot 9$ & $7 \cdot 7$ & $7 \cdot 2$ \\
\hline 7003 & & $1 \cdot 6 \mathrm{E}-15$ & P10111 & $16 \cdot 2$ & $18 \cdot 2$ & $7 \cdot 9$ & $7 \cdot 8$ \\
\hline 7209 & & $4 \cdot 2 \mathrm{E}-18$ & $\mathrm{P} 11348$ & $27 \cdot 5$ & $25 \cdot 5$ & $8 \cdot 1$ & $7 \cdot 7$ \\
\hline $7214^{*}$ & $9 \cdot 70 \mathrm{E}-06$ & $9 \cdot 9 \mathrm{E}-37$ & P08010 & $26 \cdot 6$ & $25 \cdot 7$ & $8 \cdot 3$ & $7 \cdot 3$ \\
\hline 7304 & & $3 \cdot 8 \mathrm{E}-10$ & AAH87743 & 34.4 & $36 \cdot 1$ & 8.0 & $8 \cdot 1$ \\
\hline 7602 & & $9 \cdot 8 \mathrm{E}-33$ & AAH97369 & 51.5 & $57 \cdot 0$ & 7.9 & $7 \cdot 2$ \\
\hline $7612^{*}$ & 0.00022 & $9 \cdot 1 \mathrm{E}-42$ & O70199 & $58 \cdot 0$ & 55.5 & $8 \cdot 2$ & $7 \cdot 5$ \\
\hline 7808 & 0.00067 & 8.7E-61 & A44154 & 89.5 & $86 \cdot 1$ & $8 \cdot 1$ & 7.9 \\
\hline 8312 & & $1 \cdot 1 \mathrm{E}-14$ & AAD42162 & $30 \cdot 7$ & 34.5 & $9 \cdot 2$ & $8 \cdot 8$ \\
\hline 8501 & & $3 \cdot 5 \mathrm{E}-19$ & A23126 & $49 \cdot 6$ & $47 \cdot 1$ & 8.4 & $6 \cdot 2$ \\
\hline
\end{tabular}

* Indicates identification from Matrix-assisted laser desorption/ionisation-time of flight (MALDI-TOF) spectra and confirmed using liquid chromatography/MS/MS (LCMSMS). † MALDI and LCMSMS probabilities and the corresponding Entrez code for differentially expressed proteins. The predicted mass/pl is the mass/pl is taken from the Mascot database entry. The mass/pl of the spot is that estimated from the two-dimensional gel.

\section{Discussion}

Although numerous hypotheses have been put forward to explain the importance of folic acid in reproduction, the mechanism that underlies its action remains unclear. The present study shows that there are widespread changes in both the metabolism and function of the maternal liver when the diet of pregnant rats is deficient in folic acid and the related methyl donors, methionine and choline. These findings suggest a putative mechanism related to changes in the maternal liver and lipid metabolism in particular.

It is apparent from the differentially abundant proteins involved in metabolic processes (Table 3 ) that proteins associated with folate metabolism and the methionine cycle change in response to the deficient diets. Two of the major folate binding proteins, 10-formyltetrahydrofolate dehydrogenase 
Table 3. Differentially abundant proteins involved in metabolic processes* (Data are mean pixel density with their standard errors of the mean for six rats per group)

\begin{tabular}{|c|c|c|c|c|c|c|c|c|c|}
\hline \multirow{2}{*}{$\begin{array}{l}\text { Protein } \\
\text { index } \\
\text { number }\end{array}$} & \multirow[b]{2}{*}{ Protein name } & \multirow[b]{2}{*}{ Function } & \multicolumn{2}{|c|}{ Control } & \multicolumn{2}{|c|}{$-F$} & \multicolumn{2}{|c|}{-FLMLC } & \multirow[b]{2}{*}{ Fpr } \\
\hline & & & Mean & SEM & Mean & SEM & Mean & SEM & \\
\hline 4501 & Methionine adenosyltransferase I, $\alpha$ & met cycle & $841^{\mathrm{a}}$ & 231 & $3099^{\mathrm{b}}$ & 454 & $2340^{\mathrm{ab}}$ & 791 & 0.035 \\
\hline 4809 & Formyltetrahydrofolate dehydrogenase & met cycle & $252^{\mathrm{a}}$ & 42 & $126^{b}$ & 29 & $163^{\mathrm{ab}}$ & 32 & 0.080 \\
\hline 5801 & Dimethylglycine dehydrogenase & met cycle & $569^{\mathrm{a}}$ & 33 & $354^{b}$ & 51 & $254^{\mathrm{b}}$ & 40 & $<0.001$ \\
\hline 6506 & Adenosylhomocysteinase & met cycle & $1144^{\mathrm{ab}}$ & 366 & $1715^{\mathrm{a}}$ & 226 & $833^{b}$ & 92 & 0.074 \\
\hline 3716 & Glycyl-tRNA synthetase & protein/aa metab & $352^{\mathrm{a}}$ & 24 & $348^{\mathrm{a}}$ & 31 & $702^{\mathrm{b}}$ & 49 & $<0.001$ \\
\hline 4703 & Seryl-aminoacyl-tRNA synthetase 1 & protein/aa metab & $267^{\mathrm{a}}$ & 26 & $371^{\mathrm{a}}$ & 47 & $541^{\mathrm{b}}$ & 37 & $<0.001$ \\
\hline 3002 & DOPDD-dopachrome tautomerase & tyrosine metab & 284 & 17 & 347.1 & 75 & 374 & 28 & 0.410 \\
\hline 5410 & 4-hydroxyphenylpyruvate dioxygenase & tyrosine metab & $2484^{\mathrm{a}}$ & 183 & $3866^{\mathrm{b}}$ & 591 & $2272^{\mathrm{a}}$ & 421 & 0.040 \\
\hline 5413 & Fumarylacetoacetase & tyrosine metab & $365^{\mathrm{ab}}$ & 102 & $561^{b}$ & 63 & $315^{\mathrm{a}}$ & 53 & 0.097 \\
\hline 6508 & Homogentisate 1, 2-dioxygenase & tyrosine metab & $791^{\mathrm{a}}$ & 73 & $753^{\mathrm{a}}$ & 34 & $477^{\mathrm{b}}$ & 64 & 0.007 \\
\hline 7209 & Dihydropteridine reductase & tyrosine metab & 1171 & 260 & 2450 & 672 & 2253 & 307 & 0.132 \\
\hline 2510 & Translation initiation factor elF-4A I & protein metab & $671^{\mathrm{a}}$ & 157 & $1205^{\mathrm{b}}$ & 177 & $1343^{b}$ & 145 & 0.032 \\
\hline 4404 & Amino acylase -1 & protein metab & $453^{\mathrm{a}}$ & 11 & $698^{\mathrm{b}}$ & 67 & $603^{\mathrm{b}}$ & 46 & 0.008 \\
\hline 4904 & Carbamoyl-phosphate synthase precursor & protein metab & $299^{a}$ & 38 & $197^{\mathrm{ab}}$ & 54 & $158^{\mathrm{b}}$ & 36 & 0.109 \\
\hline 5602 & Leucine aminopeptidase 3 & protein metab & $364^{\mathrm{a}}$ & 34 & $493^{b}$ & 34 & $442^{\mathrm{ab}}$ & 43 & $0 \cdot 100$ \\
\hline 6408 & Arginase, chain $\mathrm{A}$ & protein metab & $700^{\mathrm{ab}}$ & 118 & $963^{\mathrm{a}}$ & 191 & $430^{\mathrm{b}}$ & 70 & 0.067 \\
\hline 2407 & Fructose-1,6-bisphosphatase & energy metab & $1339^{\mathrm{a}}$ & 175 & $1875^{\mathrm{ab}}$ & 206 & $2235^{\mathrm{b}}$ & 232 & 0.035 \\
\hline 4306 & Cytosolic malate dehydrogenase. & energy metab & $2550^{\mathrm{a}}$ & 212 & $4000^{\mathrm{b}}$ & 291 & $3591^{\mathrm{b}}$ & 226 & 0.002 \\
\hline 5311 & Diacetyl/L-xylulose reductase & energy metab & $239^{\mathrm{a}}$ & 34 & $442^{b}$ & 78 & $296^{\mathrm{ab}}$ & 70 & $0 \cdot 100$ \\
\hline 5614 & Pyruvate kinase & energy metab & $263^{\mathrm{a}}$ & 93 & $332^{\mathrm{a}}$ & 73 & $639^{\mathrm{b}}$ & 65 & 0.010 \\
\hline 7304 & Glyceraldehyde-3-phosphate dehydrogenase & energy metab & $1271^{\mathrm{a}}$ & 134 & $823^{b}$ & 74 & $1310^{\mathrm{a}}$ & 101 & 0.014 \\
\hline 5711 & Phosphoglucomutase (Pgm1) & energy metab & $1044^{\mathrm{ab}}$ & 65 & $1203^{\mathrm{a}}$ & 28 & $998^{\mathrm{b}}$ & 67 & 0.064 \\
\hline 7602 & UDP-glucose pyrophosphorylase & energy metab & $422^{\mathrm{a}}$ & 49 & $957^{b}$ & 73 & $745^{\mathrm{c}}$ & 57 & $<0.001$ \\
\hline 7808 & Aconitate hydratase, mitochondrial precursor & energy metab & $212^{\mathrm{ab}}$ & 66 & $153^{\mathrm{a}}$ & 26 & $303^{b}$ & 36 & $0 \cdot 110$ \\
\hline 8501 & Phosphopyruvate hydratase (enolase) & energy metab & $565^{\mathrm{a}}$ & 116 & $196^{\mathrm{b}}$ & 51 & $215^{\mathrm{b}}$ & 49 & 0.007 \\
\hline 3607 & Hydroxymethylgluyaryl - CoA synthase-cytoplasmic & lipid metab & $210^{\mathrm{a}}$ & 19 & $50^{\mathrm{b}}$ & 33 & $103^{b}$ & 34 & 0.005 \\
\hline 4002 & Fatty acid binding protein & lipid metab & $649^{\mathrm{a}}$ & 69 & $980^{\mathrm{b}}$ & 94 & $960^{\mathrm{b}}$ & 55 & 0.011 \\
\hline 5008 & Fatty acid binding protein & lipid metab & $5042^{\mathrm{a}}$ & 239 & $5480^{\mathrm{a}}$ & 207 & $4081^{b}$ & 444 & 0.020 \\
\hline 5612 & Dihydrolipoamide dehydrogenase & lipid metab & $233^{\mathrm{a}}$ & 10 & $414^{\mathrm{b}}$ & 72 & $341^{\mathrm{ab}}$ & 75 & 0.134 \\
\hline 8312 & 3-hydroxyacyl-CoA dehydrogenase precursor & lipid metab & $2789^{\mathrm{ab}}$ & 586 & $3616^{\mathrm{a}}$ & 461 & $1742^{\mathrm{b}}$ & 259 & 0.034 \\
\hline
\end{tabular}

Data analysed by one-way ANOVA (Fpr) followed by Fischer's unprotected test. Mean values within a row with unlike superscript letters are significantly different $(P<0.05)$.

$-F$, diet deficient in folic acid; -FLMLC, diet deficient in all three key methyl donors, folic acid, choline and methionine.

${ }^{*}$ For details of diets and procedures, see Methods. 
Table 4. Differentially abundant structural proteins classified by function (Data are mean pixel density with their standard errors of the mean for six rats per group)

\begin{tabular}{|c|c|c|c|c|c|c|c|c|c|}
\hline \multirow{2}{*}{$\begin{array}{l}\text { Protein } \\
\text { index } \\
\text { number }\end{array}$} & \multirow[b]{2}{*}{ Protein name } & \multirow[b]{2}{*}{ Function } & \multicolumn{2}{|c|}{ Control } & \multicolumn{2}{|c|}{$-\mathrm{F}$} & \multicolumn{2}{|c|}{-FLMLC } & \multirow[b]{2}{*}{ Fpr } \\
\hline & & & Mean & SEM & Mean & SEM & Mean & SEM & \\
\hline 606 & Calreticulin precursor & ER & $551^{\mathrm{a}}$ & 83 & $272^{\mathrm{b}}$ & 58 & $206^{\mathrm{b}}$ & 37 & 0.006 \\
\hline 1306 & Apolipoprotein E precursor & ER & $738^{\mathrm{ab}}$ & 91 & $906^{\mathrm{a}}$ & 82 & $625^{\mathrm{b}}$ & 69 & 0.099 \\
\hline 1308 & Regucalcin & ER & $3418^{\mathrm{ab}}$ & 321 & $4600^{\mathrm{a}}$ & 664 & $2145^{\mathrm{b}}$ & 158 & 0.005 \\
\hline 1509 & Tubulin, $\beta, 2$ & ER & 230 & 30 & 441 & 157 & 388 & 40 & 0.303 \\
\hline 2713 & Annexin VI (67Kda) & $\mathrm{ER}$ & $205^{\mathrm{a}}$ & 17 & $263^{\mathrm{ab}}$ & 27 & $298^{\mathrm{b}}$ & 26 & 0.054 \\
\hline 2714 & dnaK-type molecular chaperone grp75 precursor & $\mathrm{ER}$ & $1582^{\mathrm{a}}$ & 115 & $1604^{\mathrm{a}}$ & 184 & $2291^{\mathrm{b}}$ & 232 & 0.032 \\
\hline 2815 & Transitional endoplasmic reticulum ATPase (EC 3.6.1.-) & ER & $284^{a}$ & 58 & $121^{\mathrm{b}}$ & 14 & $153^{\mathrm{b}}$ & 26 & 0.018 \\
\hline 5604 & Aldehyde dehydrogenase family 7, member $\mathrm{A} 1$ & ER & $460^{\mathrm{a}}$ & 85 & $929^{b}$ & 107 & $574^{\mathrm{ab}}$ & 132 & 0.022 \\
\hline 5607 & Aldehyde dehydrogenase 1 family, member B1 & ER & $942^{\mathrm{a}}$ & 116 & $1460^{\mathrm{ab}}$ & 129 & $1495^{\mathrm{b}}$ & 245 & 0.081 \\
\hline 7003 & Peptidylprolyl isomerase & ER & $380^{\mathrm{a}}$ & 87 & $137^{\mathrm{b}}$ & 48 & $171^{\mathrm{ab}}$ & 39 & 0.028 \\
\hline 7612 & UDP-glucose 6-dehydrogenase & ER & $235^{\mathrm{a}}$ & 36 & $279^{\mathrm{a}}$ & 45 & $471^{\mathrm{b}}$ & 34 & 0.002 \\
\hline 4801 & Vinculin (Metavinculin). & bile production & $117^{\mathrm{a}}$ & 14 & $65^{\mathrm{b}}$ & 12 & $96^{\mathrm{ab}}$ & 16 & 0.081 \\
\hline 5208 & Hydroxysteroid sulfotransferase & bile production & $810^{\mathrm{a}}$ & 82 & $800^{\mathrm{a}}$ & 104 & $420^{b}$ & 45 & 0.005 \\
\hline 5314 & 3-alpha-hydroxysteroid dehydrogenase & bile production & $609^{\mathrm{a}}$ & 79 & $555^{\mathrm{ab}}$ & 50 & $391^{\mathrm{b}}$ & 33 & 0.042 \\
\hline 1114 & Thiol Specific antioxidant & Oxidative stress & $843^{\mathrm{a}}$ & 63 & $1047^{\mathrm{ab}}$ & 115 & $1116^{\mathrm{b}}$ & 33 & 0.065 \\
\hline 2203 & Gamma-glutamylcysteine synthetase light chain & Oxidative stress & $243^{a}$ & 20 & $273^{\mathrm{ab}}$ & 56 & $390^{\mathrm{b}}$ & 58 & 0.037 \\
\hline 4106 & Retinol binding protein (serum precursor) & Oxidative stress & 240 & 23 & 214 & 77 & 344 & 22 & 0.168 \\
\hline 5205 & $\alpha$-tocopherol transfer protein (II) & Oxidative stress & $833^{a}$ & 53 & $1072^{\mathrm{b}}$ & 82 & $734^{\mathrm{a}}$ & 72 & 0.011 \\
\hline 6709 & Catalase $(E C 1.11 .1 .6)$ & Oxidative stress & $971^{\mathrm{a}}$ & 79 & $552^{\mathrm{b}}$ & 101 & $822^{\mathrm{ab}}$ & 106 & 0.032 \\
\hline 7214 & Glutathione S-transferase Mu 2 (GSTM2-2) & Oxidative stress & $1667^{\mathrm{a}}$ & 338 & $2285^{\mathrm{ab}}$ & 619 & $3329^{\mathrm{b}}$ & 417 & 0.085 \\
\hline 206 & 14-3-3 protein gamma-subtype ((Protein kinase $C$ inhibitor protein 1$)$ & Other & $490^{\mathrm{a}}$ & 23 & $790^{\mathrm{ab}}$ & 145 & $931^{\mathrm{b}}$ & 135 & 0.058 \\
\hline 2005 & Cellular retinal binding protein & Other & 280 & $56^{\mathrm{a}}$ & $105^{\mathrm{b}}$ & 11 & $167^{\mathrm{ab}}$ & 31 & 0.015 \\
\hline 2401 & Alpha-1-macroglobulin & Other & 116 & $19^{\mathrm{a}}$ & $195^{\mathrm{b}}$ & 23 & $126^{a}$ & 26 & 0.056 \\
\hline 4405 & Uroporphyrinogen decarboxylase & Other & 103 & $5^{\mathrm{a}}$ & $132 \cdot 7^{b}$ & 12 & $153^{\mathrm{b}}$ & 9 & 0.007 \\
\hline 4707 & Serum albumin precursor & Other & 3393 & 1151 & 1887 & 100 & 2430 & 162 & 0.308 \\
\hline
\end{tabular}

Data analysed by one-way ANOVA (Fpr) followed by Fischer's unprotected test. Mean values within a row with unlike superscript letters are significantly different $(P<0.05)$.

$-F$, diet deficient in folic acid; -FLMLC, diet deficient in all three key methyl donors, folic acid, choline and methionine; ER, endoplasmic reticulum.

For details of diets and procedures, see Methods. 

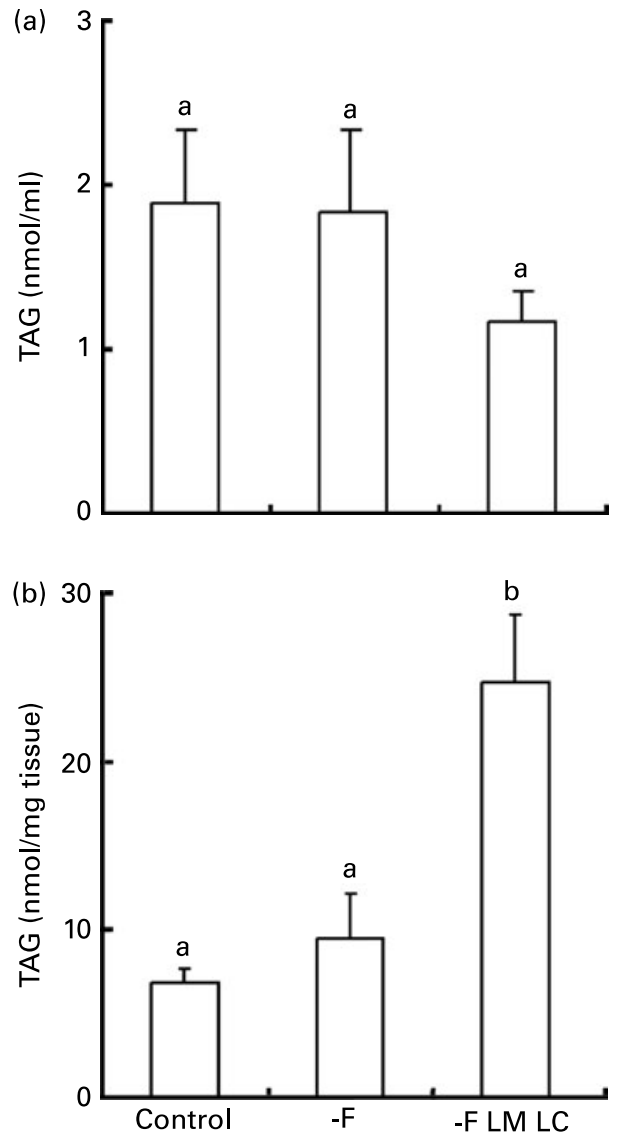

Fig. 1. TAG concentrations in maternal serum (a) and maternal liver (b). Values are means with their standard errors of the mean. Control $n 8$; diet deficient in folic acid (-F) $n$ 6; diet deficient in all three key methyl donors, folic acid, choline and methionine (-FLMLC) $n$ 7. Data analysed by one-way ANOVA followed by Fischer's unprotected test. Columns with unlike superscript letters are significantly different $(P<0.05)$. For details of diets and procedures, see Methods.

and dimethyl glycine dehydrogenase are lower in both the $-\mathrm{F}$ and -FLMLC diet groups (Table 3), a result that is consistent with the reduced hepatic folate stores in these animals ${ }^{9}$. There are also differences in the abundance of proteins associated with the methionine cycle in both groups. There is an increase in methionine adenosyl transferase and a tendency for an increase in adenosyl homocysteinease in the animals fed the -F diet, suggesting that methionine and choline compensate for the reduced availability of folates when they are available. Glycine and serine are important donors of methyl groups, which enter the methionine cycle via derivatives of folic acid. Plasma concentrations of both amino acids increase especially in the animals fed -FLMLC diets ${ }^{9}$ and the increase in the abundance of the glycyl- and seryl-t-RNA synthetases is probably a response to these changes.

Although there is no direct involvement of folic acid or its derivatives in tyrosine metabolism, a number of differentially abundant proteins are involved in the metabolism of this amino acid. Plasma tyrosine concentrations increase relative to the complete diet by $45 \%$ in animals fed the $-\mathrm{F}$ diet and decrease by $12 \%$ in animals fed the -FLMLC diets ${ }^{9}$, suggesting that these changes are having differential effects on tyrosine metabolism. Tyrosine is produced from phenylalanine via the enzyme phenylalanine hydroxylase, which requires tetrahydrobiopterin as an essential cofactor in the reaction. There are several reports of a link between folate and biopterin metabolism, possibly related to the structural and functional similarities of the folate and biopterin coenzymes $^{13}$. The present results add further circumstantial evidence to suggest that folate deficiency has an effect on tetrahydrobiopterin metabolism. As tetrahydrobiopterin is thought to be required for successful neural tube closure ${ }^{14}$, this may be a possible mechanism for an indirect effect of folate deficiency on fetal development.

Beyond the changes directly associated with the metabolism of folates and the methionine cycle, the relative abundance of proteins associated with hepatic protein, glucose and lipid metabolism is also influenced by diets deficient in folic acid and the related methyl donors (Table 3 ). The changes in protein metabolism appear to be rather non-specific and probably reflect differences in growth as the livers of animals fed the FLMLC diet are some $22 \%$ smaller than those of animals fed the complete diet. This suggests that there is a change in protein turnover that may be related to a limitation in sulphur amino acids in the animals fed the -FLMLC diet. In contrast,

Table 5. Relative mRNA levels in maternal liver*

(Values are means with their standard errors of the mean for six rats per group)

\begin{tabular}{|c|c|c|c|c|c|c|c|}
\hline \multirow{2}{*}{$\begin{array}{l}\text { Diet... } \\
\text { Gene }\end{array}$} & \multicolumn{2}{|c|}{ Control } & \multicolumn{2}{|c|}{$-F$} & \multicolumn{2}{|c|}{-FLMLC } & \multirow[b]{2}{*}{ Fpr } \\
\hline & Mean & SEM & Mean & SEM & Mean & SEM & \\
\hline Acc-1 & $17 \cdot 38^{a}$ & 0.72 & $12 \cdot 80^{b}$ & 1.24 & $13 \cdot 00^{b}$ & 0.97 & 0.009 \\
\hline L-CPT-1 & $7 \cdot 69^{a}$ & 0.44 & $9 \cdot 02^{\mathrm{a}}$ & 1.80 & $12 \cdot 45^{\mathrm{b}}$ & 1.43 & 0.037 \\
\hline C/EBP- $\alpha$ & $20 \cdot 64$ & $2 \cdot 00$ & $25 \cdot 85$ & 3.89 & 25.46 & 1.35 & 0.328 \\
\hline PPAR- $\alpha$ & $0.99^{a, b}$ & 0.06 & $0.89^{a}$ & 0.10 & $1 \cdot 26^{b}$ & 0.08 & 0.013 \\
\hline PPAR- $\gamma$ & $0.29^{a}$ & 0.04 & $0.34^{\mathrm{ab}}$ & 0.04 & $0.46^{b}$ & 0.04 & 0.031 \\
\hline SREBP-1C & $1.02^{a}$ & 0.18 & $0.51^{\mathrm{b}}$ & 0.15 & $0.57^{b}$ & 0.08 & 0.049 \\
\hline Gadd153 & 34.98 & $2 \cdot 70$ & $31 \cdot 60$ & $2 \cdot 03$ & $36 \cdot 22$ & 4.08 & 0.556 \\
\hline P57 & 23.37 & 1.39 & 23.52 & $2 \cdot 34$ & $22 \cdot 19$ & 1.67 & 0.857 \\
\hline
\end{tabular}

All data are expressed as relative expression corrected for $18 \mathrm{~S}$ rRNA. Data analysed by one-way ANOVA (Fpr) followed by Fischer's unprotected test. Mean values within a row with unlike superscript letters are significantly different $(P<0.05)$.

$-F$, diet deficient in folic acid; -FLMLC, diet deficient in all three key methyl donors, folic acid, choline and methionine; Acc, acetyl CoA carboxylase; L-CPT-1, carnitine palmitoyl transferase; C/EBP- $\alpha$, CCAAT enhancer binding protein $\alpha$; SREBP-1c, sterol response element-binding protein 1c.

${ }^{*}$ For details of diets and procedures, see Methods. 
the changes in carbohydrate and lipid metabolism suggest more specific alterations, albeit to a diverse range of processes including gluconeogenesis, glycogen synthesis, cholesterol metabolism, the malate-aspartate shuttle and mitochondrial $\beta$-oxidation. In general, the changes are greater and more widespread in the animals fed the -FLMLC diet, suggesting that the multiple deficiency is exacerbating the impact on energy and lipid metabolism. However, it is difficult to make specific predictions on the metabolic effects of these changes, since, in addition to transcriptional control, the activities of several of these enzymes are tightly regulated by a combination of allosteric effectors and phosphorylation.

The increase in hepatic TAG that occurs in the animals fed the -FLMLC diets is clearly associated with the changes in energy metabolism. Excessive synthesis and reduced oxidation of fat appear to cause steatosis in both alcoholic liver injury and non-alcoholic fatty liver disease ${ }^{15}$. However, there is no evidence for this in the case of folate deficiency. The hepatic mRNA for the key enzyme regulating fatty acid synthesis (acetyl CoA carboxylase-1) is reduced in animals from both the $-\mathrm{F}$ and - FLMLC groups. At the same time, the mRNA for carnitine palmitoyl transferase- 1 (Table 5) the key regulator of lipid oxidation is increased in the livers of animals fed FLMLC diets but not in those fed the $-\mathrm{F}$ diet. As the mRNA levels reflect the enzyme activities ${ }^{16}$, these findings suggest a reduction in the synthesis and an increase in the oxidation of fatty acids, opposing the accumulation of TAG in the FLMLC group. It appears that the responses to the $-F$ and -FLMLC diets differ because the mRNA for PPAR- $\alpha$ and PPAR- $\gamma$, which regulate fatty acid oxidation, are only increased in the animals fed the -FLMLC diets, whereas SREBP-1c, an important regulator of hepatic lipogenesis and cholesterol synthesis ${ }^{17}$, is decreased by both $-\mathrm{F}$ and FLMLC diets. The PPAR and SREBP-1c also regulate other key metabolic enzymes and the differential abundance of several other proteins is probably due to these transcription factors. For example the abundance of pyruvate kinase, which is regulated by PPAR- $\alpha^{18}$, is also increased in animals fed the -FLMLC diet.

PPAR- $\alpha$ has also been implicated in other models of hepatic steatosis, such as alcoholic fatty liver ${ }^{19}$ or diets deficient in methionine and choline ${ }^{20}$. The steatosis caused by folate deficiency during pregnancy appears to differ from other forms of steatosis, in that there is no increase in markers of liver damage such as elevated serum aspartate aminotransferase and alanine aminotransferase or an increase in the expression of gadd153, p57 and CCAAT enhancer binding protein- $\alpha$. This suggests that although there is an increase in the hepatic TAG content, it is not sufficient to induce cell damage. The metabolic changes initiated by activation of SREBP and PPAR appear to be sufficient to minimise the accumulation of lipid and this may in turn prevent the damage to cells that is responsible for initiating the pathological changes seen in models of alcoholic liver injury ${ }^{21}$.

Since production and oxidation of fatty acids does not account for the accumulation of TAG, the hepatic steatosis in animals fed the -FLMLC diet is probably caused by a reduced rate of TAG export. Evidence for this comes from the differential abundance of a number of proteins associated with the secretion of LDL, including fatty acid binding proteins, calreticulin and the apoB precursor. All of these proteins are associated with intracellular lipid handling and export via the endoplasmic reticulum and Golgi apparatus. There is also a change in the abundance of the transitional endoplasmic reticulum ATPase (down regulated in both $-\mathrm{F}$ and -FLMLC), which transports vesicles within the cell via the cytoskeleton ${ }^{22}$. All of these changes would be consistent with a reduced rate of TAG export leading to steatosis in the animals fed the -FLMLC diet. Furthermore, the steatosis caused by methyl-deficient diets is similar to that found in animals with a deletion of the PEMT gene ${ }^{23}$. As phosphatidyl choline produced by PEMT is used for the production of lipoproteins, it is a strong candidate for the underlying target of folate deficiency. The PEMT enzyme is localised in a subfraction of endoplasmic reticulum membranes, known as mitochondria-associated membranes ${ }^{24}$, where it is a major user of $\mathrm{S}$-adenosyl methionine ${ }^{23,25}$. The change in the abundance of the dnaK type molecular chaperone glucose regulated protein 75 precursor is evidence for a change in these membranes, as it has been suggested that this protein is associated with mitochondrial import, antioxidant defence and lipid metabolism ${ }^{26}$

In addition to lipoprotein production, significant amounts of the phosphocholine produced de novo by PEMT are used for the production and secretion of phosphatidic acid, lyso-phosphatidyl choline and taurocholic acid found in bile ${ }^{27}$. The down regulation of vinculin, a cytoskeletal protein specifically associated with bile canniculus ${ }^{28}$, and two proteins associated with bile production, 3- $\alpha$ hydroxysteroid dehydrogenase and hydroxysteroid sulphotransferase ${ }^{29}$, suggest that bile production is reduced in animals fed the $-\mathrm{F}$ and - FLMLC diets. Reduced bile production or secretion may have widespread effects, particularly on the digestion of lipids and the bile acid cycle. Interestingly, in addition to the production of bile acids, $3-\alpha$ hydroxysteroid dehydrogenase and hydroxysteroid sulphotransferase also have a critical role in the clearance of steroid hormones by the liver ${ }^{30-32}$. It is therefore possible that folate deficiency may have an indirect effect on systemic steroid hormone levels as a result of a reduced clearance rate.

Comparison of the soluble hepatic proteome of the pregnant female with the male rat exposed to folate-deficient $\operatorname{diets}^{33}$ shows a number of common features. These include $3-\alpha$ hydroxysteroid dehydrogenase, endoplasmic reticulumassociated proteins (glucose regulated protein 75 ) as well as changes in the antioxidant capacity. Previous studies have shown that oxidative stress is increased in folate-deficient animals and is strongly correlated to the accompanying elevation in plasma homocysteine ${ }^{33,34}$. The up regulation of $\gamma$-glutamylcysteine synthetase and glutathione S-transferase is greater in the livers of animals fed the -FLMLC diet despite the fact that plasma homocysteine concentrations are similar in animals fed $-\mathrm{F}$ and -FLMLC diets 9 . Since both groups are exposed to similar levels of homocysteine, these results suggest that the response to oxidative damage is more likely to be induced by the development of steatosis rather than a direct effect of elevated homocysteine. A similar observation of increased lipid peroxidation has been made in animals that develop a fatty liver due to a defect in VLDL secretion $^{35}$. In both cases, it appears that the fatty liver due to a defect in TAG export is associated with increased oxidative stress caused by the accumulation of TAG. 
The present study has shown that changes in the flux through the methionine cycle cause widespread changes in lipid metabolism and export from the maternal liver, suggesting that lipid metabolism may be a common factor linking folate status, BMI and NTD occurrence. During pregnancy, fatty acids derived from lipoprotein-associated TAG provide an important source of lipid for the developing fetus ${ }^{36}$. By interfering with the export of lipoprotein, folate deficiency may disrupt this supply. It is striking that NTD and other developmental anomalies are more common in infants born to obese women ${ }^{37}$. A BMI $>30$ doubles the risk of having a child with a NTD compared with normal-weight women and, critically, this increased risk does not appear to be modified by folic acid supplementation ${ }^{38}$. Obese individuals frequently have steatotic livers, suggesting a possible link between hepatic lipid metabolism and NTD. The close interrelationship between folates, the methionine cycle and fat metabolism suggest that high-fat, low-folate diets, typical of those found in Western Europe, may be delivering a double blow to the delivery of essential lipids to the developing fetus.

\section{Acknowledgements}

This work was supported by the Scottish Executive Environment and Rural Affairs Department as part of the core funds of the Rowett Research Institute and by the European Union Sixth Framework programme EARNEST (CT-2005-007036). Christopher Maloney is supported by a cooperative agreement from the NIH (U01 HD044638) as a component of the NICHD Cooperative Program on Female Health and Egg Quality. We express our thanks to staff from Bioresources Unit for animal care and to Dr C. Mayer (Biomathematics and Statistics, Scotland) for advice on the statistical analysis.

\section{References}

1. Butterworth CE \& Bendich A (1996) Folic acid and the prevention of birth defects. Annu Rev Nutr 16, 73-97.

2. Stover PJ (2004) Physiology of folate and vitamin B12 in health and disease. Nutr Rev 62, S3-S12.

3. Copp AJ, Greene NDE \& Murdoch JN (2003) The genetic basis of mammalian neurulation. Nat Rev Genet 4, 784-793.

4. Tamura T \& Picciano MF (2006) Folate and human reproduction. Am J Clin Nutr 83, 993-1016.

5. Relton CL, Pearce MS \& Parker L (2005) The influence of erythrocyte folate and serum vitamin B12 status on birth weight. Br J Nutr 93, 593-599.

6. Scholl TO \& Johnson WG (2000) Folic acid: influence on the outcome of pregnancy. Am J Clin Nutr 71, 5 Suppl., 1295S-1303S.

7. Stead LM, Brosnan JT, Brosnan ME, Vance DE \& Jacobs RL (2006) Is it time to reevaluate methyl balance in humans? Am $J$ Clin Nutr 83, 5-10.

8. American Institute of Nutrition (1977) Report of the American Institute of Nutrition ad hoc committee on standards for nutritional studies. $J$ Nutr 107, 340-348.

9. Maloney CA, Hay SM \& Rees WD (2007) Folate deficiency during pregnancy impacts on methyl metabolism without affecting global DNA methylation in the rat fetus. Br J Nutr 97, 1090-1098.

10. de Roos B, Duivenvoorden I, Rucklidge G, Reid M, Ross K, Lamers RJ, Voshol PJ, Havekes LM \& Teusink B (2005) Response of apolipoprotein $\mathrm{E}^{*} 3$-Leiden transgenic mice to dietary fatty acids: combining liver proteomics with physiological data. FASEB $J$ 19, 813-815.

11. Drew JE, Padidar S, Horgan G, Duthie GG, Russell WR, Reid M, Duncan G \& Rucklidge GJ (2006) Salicylate modulates oxidative stress in the rat colon: A proteomic approach. Biochem Pharmacol 72, 204-216.

12. Maloney CA, Lilley C, Cruickshank M, McKinnon C, Hay SM \& Rees WD (2005) The expression of growth-arrest genes in the liver and kidney of the protein-restricted rat fetus. Br J Nutr $\mathbf{9 4}$, $12-18$.

13. Lucock M, Yates Z, Hall K, Leeming R, Rylance G, MacDonald A \& Green A (2002) The impact of phenylketonuria on folate metabolism. Mol Genet Metab 76, 305-312.

14. Nachmany A, Gold V, Tsur A, Arad D \& Weil M (2006) Neural tube closure depends on nitric oxide synthase activity. J Neurochem 96, 247-253.

15. Reddy JK \& Sambasiva Rao M (2006) Lipid metabolism and liver inflammation. II. Fatty liver disease and fatty acid oxidation. Am J Physiol Gastrointest Liver Physiol 290, G852-G858.

16. Kim TS \& Freake HC (1996) High carbohydrate diet and starvation regulate lipogenic mRNA in rats in a tissue-specific manner. J Nutr 126, 611-617.

17. Brown MS \& Goldstein JL (1997) The SREBP pathway: regulation of cholesterol metabolism by proteolysis of a membranebound transcription factor. Cell 89, 331-340.

18. Xu J, Christian B \& Jump DB (2006) Regulation of rat hepatic L-pyruvate kinase promoter composition and activity by glucose, n-3 polyunsaturated fatty acids, and peroxisome proliferator-activated receptor-\{alpha\} agonist. J Biol Chem 281, $18351-18362$.

19. You M \& Crabb DW (2004) Recent advances in alcoholic liver disease II. Minireview: molecular mechanisms of alcoholic fatty liver. Am J Physiol Gastrointest Liver Physiol 287, G1-G6.

20. Ip E, Farrell GC, Robertson G, Hall P, Kirsch R \& Leclercq I (2003) Central role of PPARalpha-dependent hepatic lipid turnover in dietary steatohepatitis in mice. Hepatology 38, 123-132.

21. Kaplowitz N \& Ji C (2006) Unfolding new mechanisms of alcoholic liver disease in the endoplasmic reticulum. $J$ Gastroenterol Hepatol 21, s3, S7-S9.

22. Fisher EA \& Ginsberg HN (2002) Complexity in the secretory pathway: The assembly and secretion of apolipoprotein B-containing lipoproteins. J Biol Chem 277, 17377-17380.

23. Noga AA \& Vance DE (2003) A gender-specific role for phosphatidylethanolamine $\mathrm{N}$-methyltransferase-derived phosphatidylcholine in the regulation of plasma high density and very low density lipoproteins in mice. J Biol Chem 278, 21851-21859.

24. Cui Z, Vance JE, Chen MH, Voelker DR \& Vance DE (1993) Cloning and expression of a novel phosphatidylethanolamine $\mathrm{N}$ - methyltransferase. A specific biochemical and cytological marker for a unique membrane fraction in rat liver. $J$ Biol Chem 268, 16655-16663.

25. Shields DJ, Lehner R, Agellon LB \& Vance DE (2003) Membrane topography of human phosphatidylethanolamine Nmethyltransferase. J Biol Chem 278, 2956-2962.

26. Wadhwa R, Taira K \& Kaul SC (2002) An Hsp70 family chaperone, mortalin/mthsp70/PBP74/Grp75: what, when, and where? Cell Stress Chaperones 7, 309-316.

27. Sehayek E, Wang R, Ono JG, et al. (2003) Localization of the PE methylation pathway and SR-BI to the canalicular membrane: evidence for apical PC biosynthesis that may promote biliary excretion of phospholipid and cholesterol. J Lipid Res 44, 1605-1613.

28. Roman LM \& Hubbard AL (1983) A domain-specific marker for the hepatocyte plasma membrane: localization of leucine aminopeptidase to the bile canalicular domain. JCell Biol 96, 1548-1558.

29. Tsukada N, Ackerley CA \& Phillips MJ (1995) The structure and organization of the bile canalicular cytoskeleton with 
special reference to actin and actin-binding proteins. Hepatology 21, 1106-1113.

30. Falany CN (1997) Enzymology of human cytosolic sulfotransferases. FASEB J 11, 206-216.

31. Baumgartner U, Baier P, Birke O \& Farthmann EH (2003) The 3alpha-hydroxy-steroid-dehydrogenase-mRNA and protein are more prevalent in pericentral than in periportal hepatocytes. Eur J Gastroenterol Hepatol 15, 509-513.

32. Penning TM, Jin Y, Steckelbroeck S, Lanisnik Rizner T \& Lewis M (2004) Structure-function of human 3[alpha]-hydroxysteroid dehydrogenases: genes and proteins. Mol Cell Endocrinol 215, 63-72.

33. Chanson A, Sayd T, Rock E, Chambon C, Sante-Lhoutellier V, Potier de Courcy G \& Brachet P (2005) Proteomic analysis reveals changes in the liver protein pattern of rats exposed to dietary folate deficiency. $J$ Nutr $\mathbf{1 3 5}, 2524-2529$.
34. Huang RF, Hsu YC, Lin HL \& Yang FL (2001) Folate depletion and elevated plasma homocysteine promote oxidative stress in rat livers. J Nutr 131, 33-38.

35. Lin X, Chen Z, Yue P, Averna MR, Ostlund RE Jr, Watson MA \& Schonfeld G (2006) A targeted apoB38.9 mutation in mice is associated with reduced hepatic cholesterol synthesis and enhanced lipid peroxidation. Am J Physiol Gastrointest Liver Physiol 290, G1170-G1176.

36. Lindegaard MLS, Olivecrona G, Christoffersen C, Kratky D, Hannibal J, Petersen BL, Zechner R, Damm P \& Nielsen LB (2005) Endothelial and lipoprotein lipases in human and mouse placenta. J Lipid Res 46, 2339-2346.

37. King JC (2006) Maternal obesity, metabolism, and pregnancy outcomes. Аnпи Rev Nutr 26, 271-291.

38. Anthony RS (2006) Teratology public affairs committee position paper: Maternal obesity and pregnancy. Birth Defects Res A: Clin Mol Teratol 76, 73-77. 